\title{
RAPID SERIAL ANGIOGRAPHY: FURTHER EXPERIENCE
}

\author{
BY
}

\section{PETER H. SCHURR and INGMAR WICKBOM}

\author{
From the Departments of Neurosurgery and Radiology, the Radcliffe Infirmary, Oxford
}

In 1949 a preliminary report on a method of rapid serial angiography of the cerebral vessels was published from this hospital by Curtis (1949). When he returned to Australia we took over the work on this subject, and in this paper we report our observations and an analysis of the serial angiograms which have been made here during the two years in which this method has been used.

\section{Apparatus}

A brief description of the machine (Fig. 1) was given in the earlier paper on this subject (Curtis, 1949), and this apparatus has been used for all the cases without modification. It was devised by Dr. E. Schuster and it enables exposures to be made at three variable speeds up to 2 frames per second. Intensifying screens are employed, and the images, each 10 in. wide by 8 in. long, are projected on to a strip of film $20 \mathrm{ft}$. in length.

An antero-posterior projection has been obtained by rotating the head, but this is unsatisfactory because it is difficult to get accurate alignment, and the lateral projection only is in general use. This has been found to be adequate for most purposes when supplemented by antero-posterior views taken by the standard three-film technique (Curtis, 1951). After injection of the vertebral artery, however, it would be particularly useful if rapid serial angiograms could be taken in the half-axial projection, which provides more information than does the lateral vertebral angiogram. Several models of the machine have been made by Dr. Schuster for other purposes in which this projection is required, and it would be a relatively simple matter to adapt one of these for cerebral angiography.*

There are one or two minor disadvantages, of a technical nature, which depend on the difficulty of developing a long strip of film. As was described in the preliminary report (Curtis, 1949), the film is

* Messrs. General Radiological Limited hope to manufacture a unit base on a later design by Dr. Schuster in collaboration with the Nuffield Institute for Medical Research. This model is intended for general use but is readily adaptable for cerebral angiography in any projection ; it was
Radiology in 1950 . wound on to a spiral holder of a type similar to that used for aerial photography. Owing to the impossibility of winding wet film back on to the holder once it has been taken off, the process of development, fixation, and washing must be completed before the film is removed. This involves a delay of about an hour before the wet film can be inspected. Apart from the necessity of waiting this time before a diagnosis can be reached, a more serious problem is that the angiogram cannot be repeated on the same occasion if for any reason there should have been a fault in the injection or radiography. Also, in order to study the wet film special fixtures for viewing it are necessary. These are usually not available in the operating theatre, so that if the film is required for immediate exhibition during an operation a further delay is caused.

\section{Irradiation}

We have considered the possibility that the amount of irradiation received as a result of a large number of rapidly repeated exposures might be harmful to the patient. By means of accurate measurements the dose has been found not to exceed $20.8 \mathrm{r}$ for the maximum number of exposures, which is 25 per film. In practice such a large number of frames is seldom necessary, since most of the contrast has usually passed through the cerebral vessels in eight seconds* as will be shown (Fig. 2). The machine can be stopped automatically after any given number of exposures. The procedure may therefore be repeated once with safety should it be necessary, and if the likelihood of this is envisaged a short film can be taken.

These measurements serve nevertheless to emphasize the need for really efficient protection of the operators, particularly the person responsible for making the injection. A wide curtain of leadrubber sheeting $1 \mathrm{~mm}$. thick has been found adequate, and with this the maximum dosage for the operator is $0.005 \mathrm{r}$ during 25 exposures.

* Throughout this paper circulation times quoted have been measured from the beginning of the injection. 
FIG. 1.-A. Rapid serial angiographic apparatus. The power unit is seen below, with a connecting rod leading to the cassette mounted on a Lysholm-Schönander table. The exposure, the spool drive, and the movable intensifying screens are synchronized and operated electrically. The protecting screens and table have been removed for greater clarity.

B. Closer view of power unit. (1) motor; (2) automatic brake and device for altering the pre-set number of exposures ; (3) gear lever ; (4) electromagnet, operating (5) clutch ; (6) starting switch.

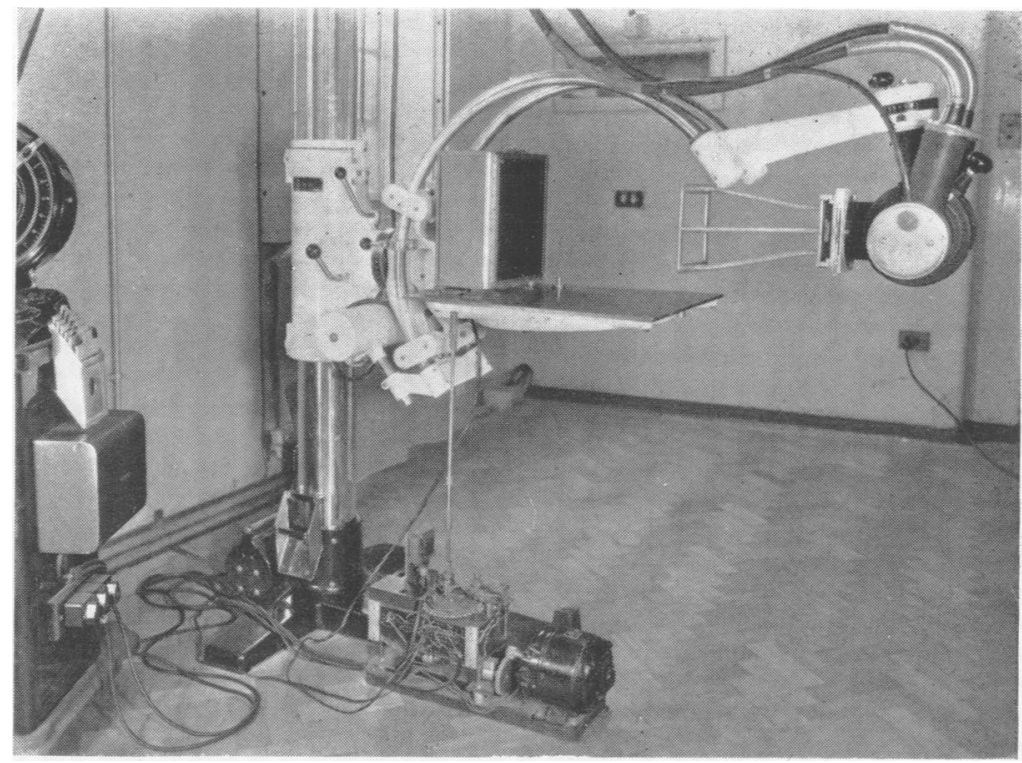

Fig. 1A

\section{Case Material}

The material consists of 109 serial angiograms from 74 patients. Twenty-one of these angiograms were considered to be within normal limits. The diagnoses of the cases reviewed are analysed in Table I. In a recent paper Curtis (1951) mentioned his observations on some of these films.

Serial angiography is as a rule only employed in those cases in which it is expected that more information will be obtained than by the standard technique. Economy also dictates careful selection, as the cost of a full film of 25 exposures is seven times that of the film used in the standard technique. In the six-month period, October, 1950, to March, 1951, 131 angiographic examinations were made at the Radcliffe Infirmary, for which the rapid serial method was employed in 19.

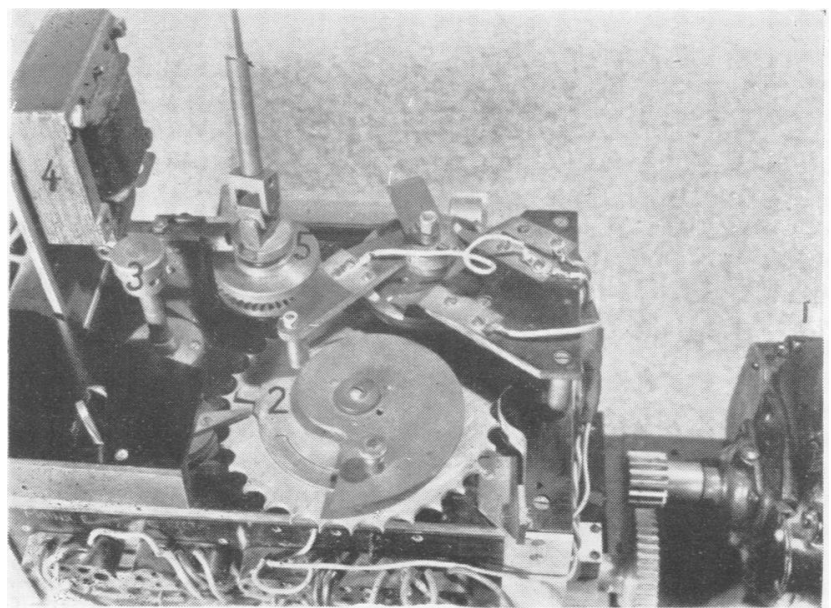

FIG. 1B
We intend to discuss only those points which have arisen as a result of our study of the serial angiograms taken in the last two years, as the more general aspects of the subject have been frequently described elsewhere.

\section{The Normal Cerebral Circulation}

The "normal" material consists of those angiograms in which no displacement of vessels or other abnormality was visible in any part of the film, and in which the diagnosis of the case was such that we did not consider it likely that the part of the circulatory system being studied was affected to any significant extent by the disease process (Table II).

Variations in Circulation Time.-In order to facilitate comparison, the circulation times in the 21 normal cases have been tabulated diagrammatically in Fig. 2. The most striking feature will be seen to be the large variation between the cases. When the durations of the venous phases are compared it is seen that these varied in length from two seconds to more than nine and a half seconds. The arterial phases were more consistent and varied from one and one third to three and one third seconds.

Of course, the cerebral circulation is influenced in many ways by present methods of angiography ; it is not, therefore studied under physiological conditions. The main technical factors which may affect the record of the circulation that is obtained are as follows. 
TABLE I

CASE MATERIAL

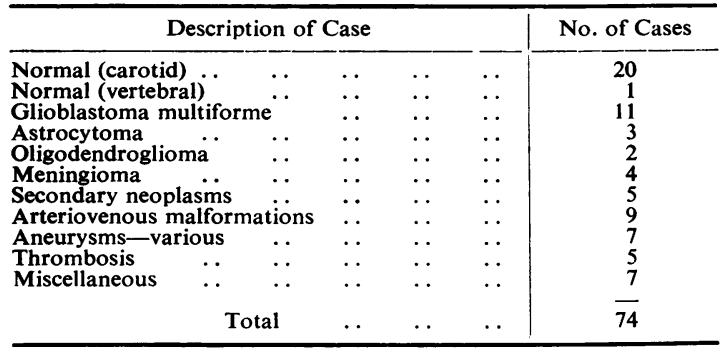

TABLE II

DIAGNOSES OF CASES WITH " NORMAL" ANGIOGRAMS

\begin{tabular}{|c|c|c|c|}
\hline Case & Diagnosis & Case! & Diagnosis \\
\hline $\begin{array}{r}1 \\
2 \\
3 \\
4 \\
4 \\
5 \\
6 \\
7 \\
8 \\
9 \\
10\end{array}$ & $\begin{array}{l}\text { Focal epilepsy } \\
\text { Otitic hydrocephalus } \\
\text { Thrombosis in opposite } \\
\text { hemisphere } \\
\text { Subarachnoid haemorrhage } \\
\text { Meningitis (pyogenic) } \\
\text { Penetrating parietal injury } \\
\text { Subarachnoid haemorrhage } \\
\text { Subarachnoid haemorrhage } \\
\text { Suspected tumour } \\
\text { Thrombosis in opposite } \\
\text { hemisphere }\end{array}$ & $\begin{array}{l}11 \\
12 \\
13 \\
14 \\
15 \\
16 \\
17 \\
18 \\
19 \\
20 \\
21\end{array}$ & $\begin{array}{l}\text { Focal epilepsy } \\
\text { Secondary carcinoma in } \\
\text { cerebellum } \\
\text { Cerebral arteriosclerosis } \\
\text { Focal epilepsy } \\
\text { Migraine } \\
\text { Senile cerebral degeneration } \\
\text { Migraine } \\
\text { Focal epilepsy. } \\
\text { Focal epilepsy } \\
\text { Focal epilepsy } \\
\text { Subarachnoid haemorrhage }\end{array}$ \\
\hline
\end{tabular}

The Puncture.-The size of the lumen of the needle influences the rate at which the contrast substance can be injected, and the site at which the needle is inserted into the carotid system makes a considerable difference to the angiogram obtained. If the injection is made into the internal carotid artery, all the contrast substance is delivered into the cerebral circulation. If, on the other hand, the injection is made into the common carotid artery, the proportion of contrast medium which is distributed between the external and internal carotid circulations is variable. The predominant course taken by the opaque fluid is governed to some extent by the direction in which the bevelled end of the needle is pointed. The flow of the contrast substance is also impeded if the needle does not lie fully within the lumen of the vessel, and we have omitted cases from consideration in which doubt existed about the freedom of flow through the needle. If the puncture is difficult the artery may be compressed by extravasated blood or put into spasm, and direct stimulation of the carotid sinus is also a possibility.

The Contrast Substance.-We have no accurate details of the action of diodone*, which was the contrast substance used in all these cases, on the cerebral blood vessels. The flushing of the face, often preceded by transient pallor, and injection of

* Pyelosil, Glaxo. the conjunctiva, are well known effects on extracerebral vessels. There is possibly some stimulation of perivascular autonomic fibres since the injection is frequently associated with pupillary dilatation.

The contractility of pial vessels has been established (Florey, 1925 ; Forbes and Wolff, 1928) and the intracerebral arteries may be expected to be affected in a similar manner to those of the extracerebral circulation. The duration of this influence may be such as to affect the circulation time when contrast substance is injected twice at the same session, but we have insufficient material to draw any definite conclusions on this point. In three cases, however, in which the serial examination was repeated after a short interval (about 20 minutes), no significant differences in the circulation time were demonstrated. The only means of demonstrating any direct influence of the contrast substance on the circulation time would be to compare a number of angiograms in which two injections were given : for the first injection a non-irritant substance would be employed, and for the second, diodone. The only non-irritant contrast medium available at the present time is thorotrast, and we have not felt justified in using this because of its possible harmful effects.

The Injection.-The main factor affecting the duration of vascular filling is the time occupied by the injection; this of course depends on the volume of contrast substance and the speed of injection. The volume given to adults in our series was usually 10 or $12 \mathrm{ml}$, depending on the length of rubber tubing attached to the syringe, and the time occupied by the injection was about two seconds in all our cases. As the first frame is taken at the moment the injection is begun, it is possible to measure this time in the serial films by noting the disappearance of the column of contrast from the neck. The rate of injection evidently affects pressure relationships within the Circle of Willis, and on account of this some vessels occasionally take up contrast at the expense of others. We have found that when the anterior cerebral artery has failed to fill it has sometimes been due to too rapid injection of the contrast substance. Obviously, the volume injected will also influence the circulation time, and the shortest in our series (Case 2) occurred in a child aged 3 years, in whom an injection of only $6 \mathrm{ml}$. of contrast was used.

Other Factors.-The wide variations in the normal circulation time cannot be entirely explained by the technical factors of the injection, and, although conditions are manifestly far from physiological during angiography, we feel justified in concluding 
that the normal circulation time is subject to considerable variation. This is probably due partly to differences in pulse rate, pulse volume, and blood pressure. These factors may in their turn be influenced by the procedure to some degree : the patient will often be more or less upset by the investigation, with resulting increase in the pulse rate and consequently a more rapid circulation time. As shown by Curtis (1951), the blood pressure may be raised after injection of diodone into the carotid arteries. As a further cause of variations among apparently normal circulation times, the possibility of arteriovenous or intervenous anastomotic channels in the brain, similar to those described in the kidney (Trueta, Barclay, Daniel, Franklin, and Prichard, 1947) and liver (Daniel and Prichard, 1951), should also be considered.

The Circulatory Phases.-We find that there is no direct proportion between the length of the arterial phase and that of the venous phase (Fig. 2). The capillary phase was seen in comparatively few serial angiograms with frames at intervals of $\frac{2}{3}$ second. This apparent overlap of the various parts of the sequence is presumed to be partly due to the volume

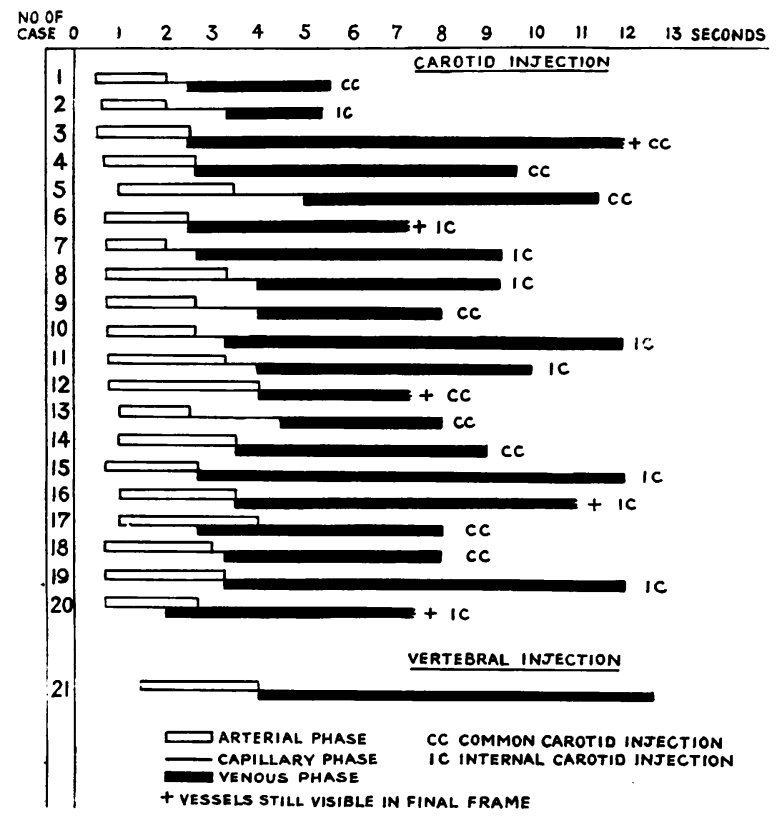

FIG. 2.-Circulation times in 21 normal serial angiograms. About $10 \mathrm{ml}$. diodone (allowing for the volume within the tubing) were injected for each angiogram, except in Cases $2(6 \mathrm{ml}),$.5 , and $12(8 \mathrm{ml}$.). Cases 2 and 17 were examined under general anaesthesia. Case 2 was 3 years old, the remainder were adult (19-52 years). The inferior longitudinal sinus is not included in measurements of the venous phase. The complete angiogram of Case 10 was illustrated in the preliminary report (Curtis, 1949). of the contrast substance, but another important point is that the capillary phase may not take place over the whole brain at the same moment. These remarks are not fully in agreement with the views of Moniz (1940), but some differences may be due to the fact that he used thorotrast for his injections. We do not therefore feel that it is possible at the present time to draw any hard and fast conclusions about the duration of the phases of the cerebral circulation. It will be appreciated that it is impossible to get a film of any particular phase, other than the arterial one, with any degree of reliability using only three frames.

The Sequence of Venous Filling.-Curtis (1951) stated that in the venous phase " the deep cerebral veins and certain superficial veins around the anterior part of the temporal lobe and Sylvian point are the first to be seen ". This was true in 15 of our 20 normal serial carotid angiograms, but in the remainder the inferior longitudinal sinus was

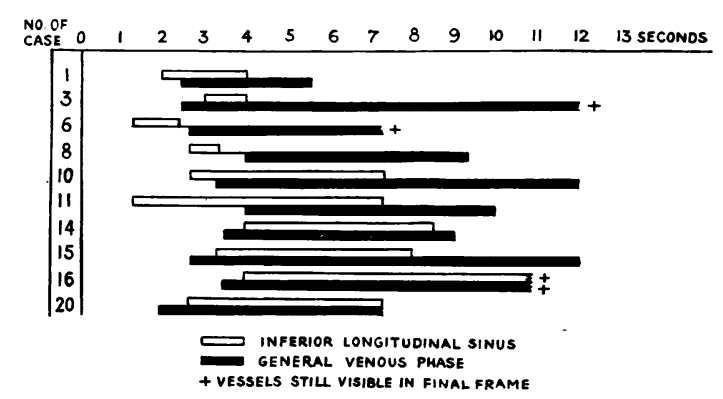

FIG. 3.-The duration of filling of the inferior longitudinal sinus has been compared with that of the remainder of the cerebral venous channels. The sinus was visible in 10 cases out of 20 , and was the first venous channel to appear in five cases out of 10 .

the first venous channel to be seen. In some films it appeared during the arterial phase when the arteries were still well filled by the contrast substance. The relationship of filling of the inferior longitudinal sinus to the filling of the other superficial and deep veins is shown in Fig. 3. It is a curious fact that in 10 of the 20 cases this sinus was not visible at any stage.

The deep (internal) cerebral veins are usually seen to fill in the same frame as the superficial cersbral veins, or often a little later (Fig. 4).

Our experience does not confirm Curtis's staiement (1951) that the deep veins are usually the first to empty. In our 20 cases they emptied first in three, last in five, and in the others at about the same time as the superficial veins or at a time which was indeterminable on account of the presence of contrast in both sets of veins in the last frame (Fig. 4). 


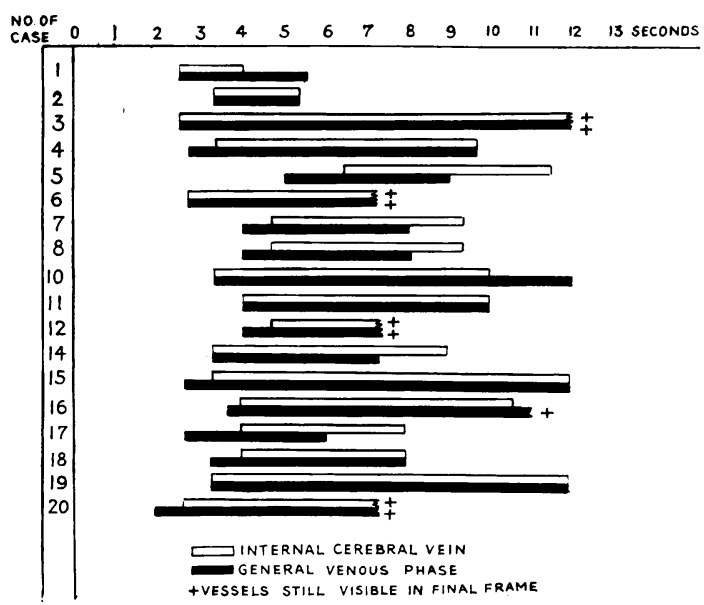

FIG. 4.-Comparison of the duration of filling of the internal cerebral vein with that of the remaining venous channels, excluding the inferior longitudinal sinus. Cases 9 and 13 are omitted because the internal cerebral vein was not sufficiently clearly seen in these angiograms to allow conclusions to be drawn. This vessel appears at the same time, or just after the other venous channels, and in the 14 cases in which it can be followed through it disappeared first in three, last in five, and together with the remaining vessels in six.

\section{The Choroidal Plexus of the Eye}

During the study of these rapid serial angiograms a crescentic line was observed within the orbit produced by contrast substance in the choroidal plexus of the eye (Schurr, 1951). This appearance was found in 11 of the 20 normal carotid angiograms, and it was visible between the average times of two and five and a half seconds (Fig. 5). It is probably produced mainly by venous channels, since it usually begins to appear when the ophthalmic

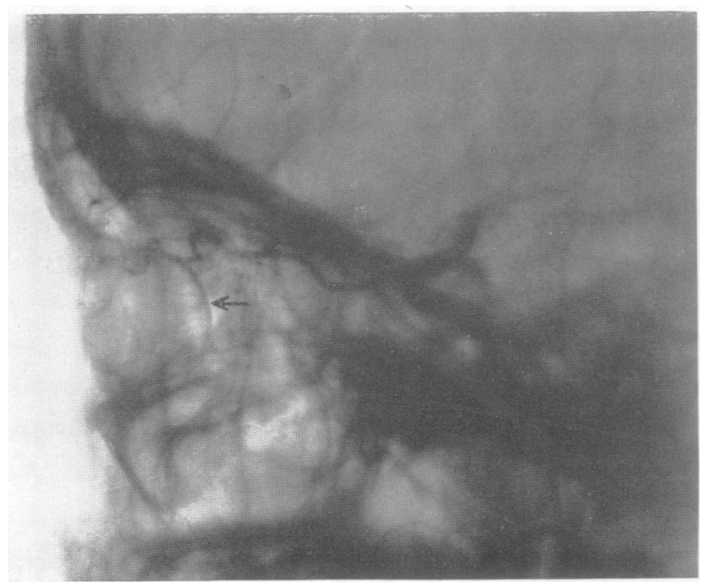

$\begin{array}{ccc}\text { FIG. 5A } & \text { FIG. 5B } \\ \text { Fig. 5.-A. } 5 \frac{1}{3} \text { seconds. } & \text { B. } 6 \text { seconds. Frames from serial angiograms of two cases showing filling of the choroidal plexus of the eye }(\leftarrow)\end{array}$ artery is fading, and it remains for about half the duration of the venous phase of the cerebral circulation. The contrast seems to appear first at a point just above the meridian of the globe. It develops as a fine crescent with clear outlines, and then gradually fades away. Pathological appearances of the choroidal plexus have not yet been studied, but it is possible that a technique could be developed which would be of use in the demonstration and differential diagnosis of some orbital and ocular neoplasms.

\section{The Pathological Circulation}

Tumour Diagnosis. - It is a matter of common experience that it is easier to come to a conclusion about a diagnosis if there are several slightly different films on which to base an opinion than if there are only one or two. In these circumstances a serial film may be a great help. When doubt exists about the presence or absence of a vessel, or whether it is a vein or an artery, the matter can usually be resolved by watching its gradual appearance and disappearance in successive frames of a serial angiogram.

It happens on rare occasions that a tumour circulation is only visible for a brief period, and with only three films it is possible to miss the whole or part of the abnormality.

An example is shown in Fig. 6 of a patient aged 47 in whom there were symptoms and signs which suggested that a hypernephroma, removed four years previously, had metastasized to the brain. A temporal deposit was seen in the routine series of three films, but when exposures were made between the normal intervals a second deposit was clearly seen in the occipital region; this was scarcely visible in the first series and was certainly not

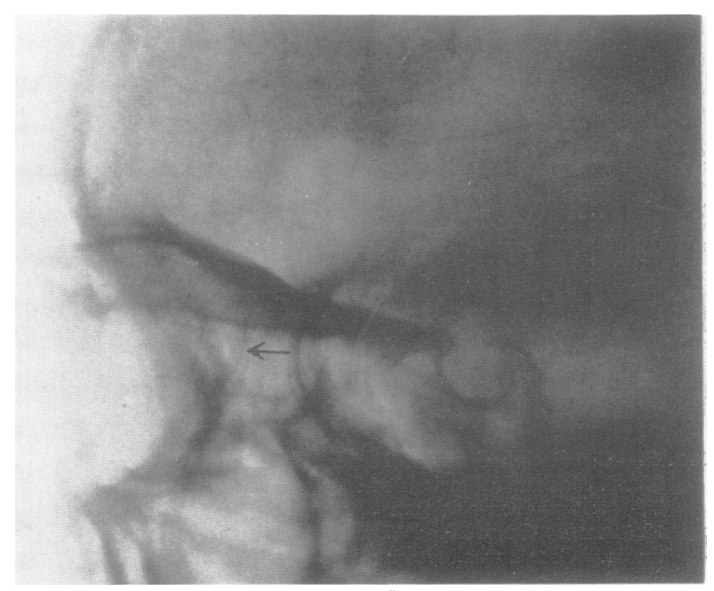

Fig. 5B 


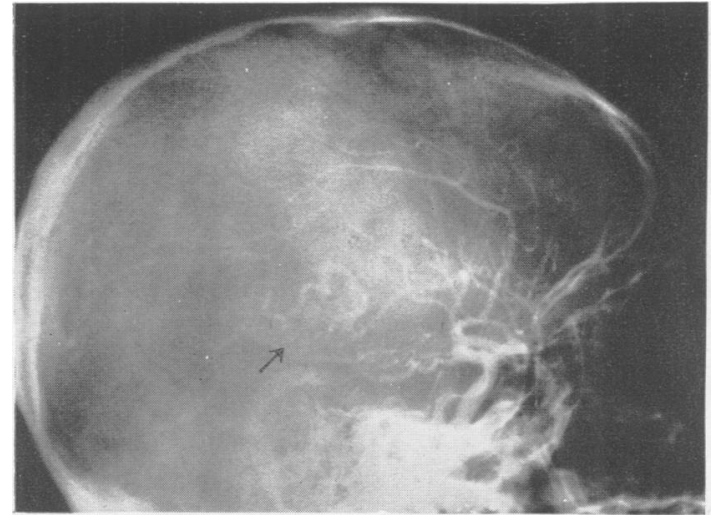

Fig. 6A

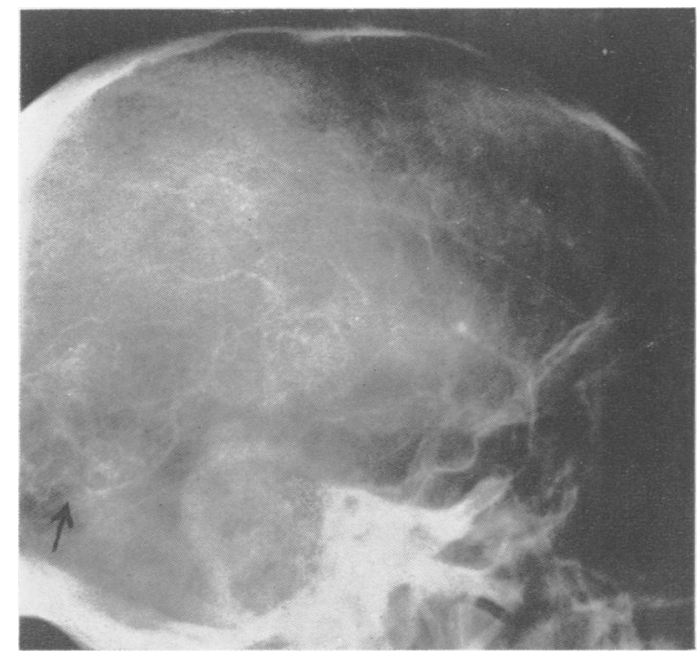

Fig. 6B

A. Arteriogram (2 seconds) from a standard series showing the tumour circulation of a metastatic hypernephroma in the temporo-parietal region $(\uparrow)$.

B. Arteriogram (1 second) from a series of films taken between the standard times showing another deposit in the occipital region ( $\uparrow)$. This was not sufficiently clearly seen in any of the films of the first series for a diagnosis to be made.

sufficiently well defined for diagnosis. The presence of the second large deposit made operative removal impossible.

It sometimes happens that, when a tumour does not reveal a pathological circulation or does not lie in direct relation to any of the larger arteries, a diagnosis has to be based on the displacement of very small vessels. With the larger number of exposures obtained by the serial method there is greater opportunity of obtaining good films of these small channels (Fig. 7).

Malignant Gliomas.-The presence of arteriovenous fistulae in a tumour other than an arterio-

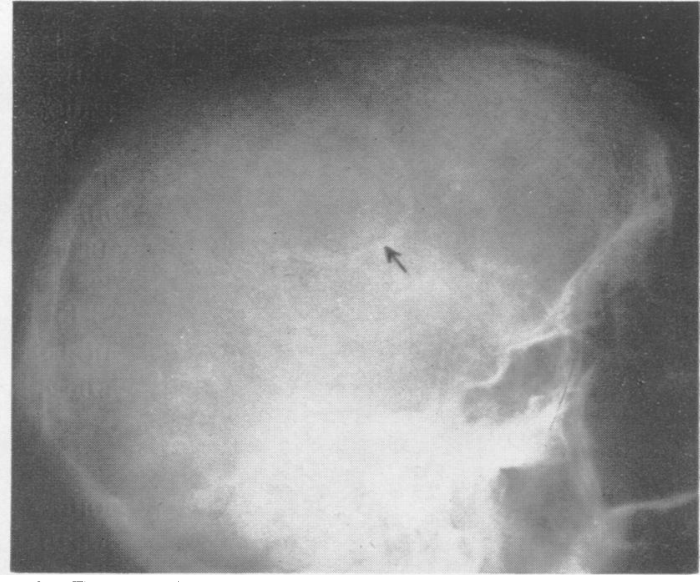

Fig. 7.-Frame at 4 seconds from a serial angiogram of a patient with a fronto-parietal glioblastoma multiforme. The localization of almost avascular tumours depends on the observation of displacements of small vessels $(\uparrow)$ which may fill for only a brief interval and are therefore more clearly seen in serial films. These vessels could not be identified in the previous frame ( $3 \frac{1}{2}$ seconds), and were invisible again three frames later (5 $\frac{1}{2}$ seconds). Retouched.

venous malformation, has come to be recognized as one of the chief radiological characteristics of malignancy in a glioma. This feature is of extreme importance in the diagnosis of a glioblastoma multiforme, and with further experience it may prove that as a criterion of malignant change it is of more value than histological examination of a smear which may happen to have been taken from a part of the neoplasm in which the original nonmalignant type of cell still exists.

The early filling of tumour veins can be accurately studied in serial angiograms, and the time of their

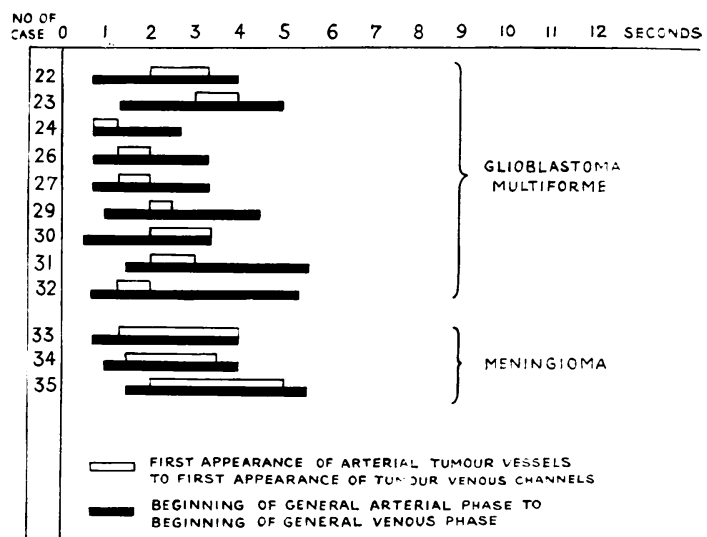

FIG. 8.-The interval between the first appearance of arterial tumour vessels and their venous efferents compared with the time between the appearance of the first arteries and the first venous channels elsewhere in the cerebral circulation. This is shown in nine cases of glioblastoma multiforme and three cases of meningioma. In two other cases of each type the tumour vessels were insufficiently clear for comparison. The rapid passage of contrast medium into the efferent vessels of the glioblastomas is clearly seen. 


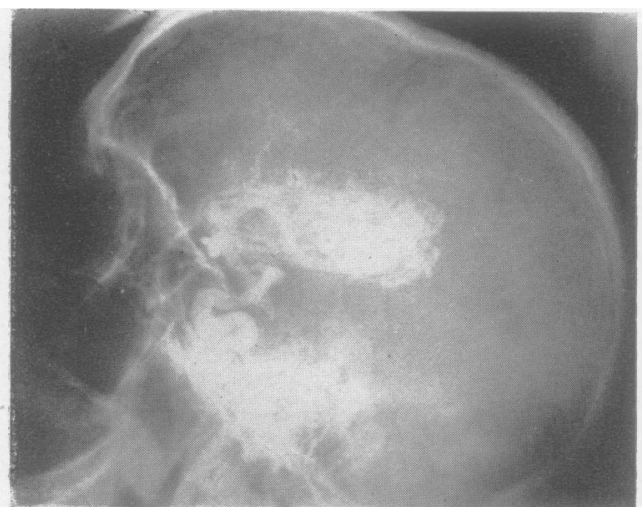

FIG. 9A

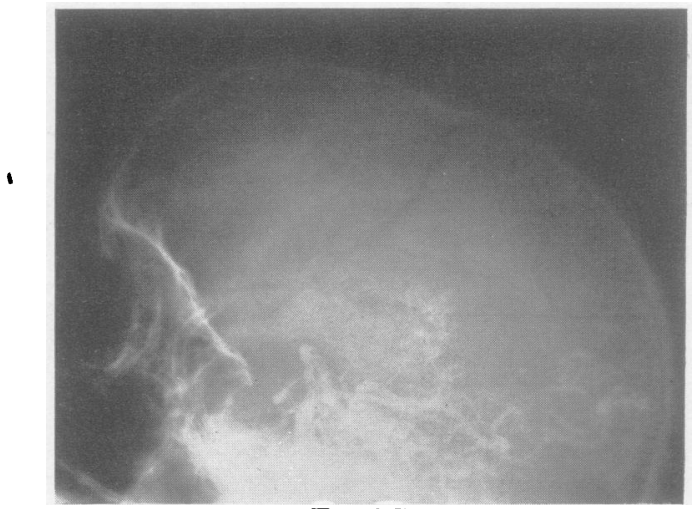

FIG. 9C

FIG. 9.-A. $1 \frac{1}{3}$ seconds. B. $2 \frac{2}{3}$ seconds.

Frames from a serial angiogram of a large arteriovenous malformation taken during an injection of the left internal carotid artery. The contrast can be seen to spread through a dense cluster of abnormal vessels, and to enter the dilated and tortuous venous efferents at an early stage. The anterior cerebra artery has not filled clearly but is just visible. The main blood flow is seen to be through the lesion.

C. 2 seconds. D. $3 \frac{1}{3}$ seconds.

Frames taken during an injection into the left vertebral artery of the same patient. The vascular abnormality is seen to be much more extensive than was first shown to be the case, different parts of the lesion being supplied by the middle and posterior cerebral arteries.

E. 6 seconds. A later film in the vertebral series shows the presence of a veil-like lake of contrast substance, lying in grossly dilated venous channels not visible after carotid injection and appearing late in the series.

appearance can be compared with that of the remainder of the cerebral venous channels. In Fig. 8 the interval between the appearance of the first arterial vessels in a tumour and the first vein leading from it is compared with the beginnings of the arterial and venous phases of the general cerebral circulation in the same case. It will be seen that the contrast passes relatively rapidly into the venous efferents of the glioblastomas, in which arteriovenous fistulae are plentiful, and with

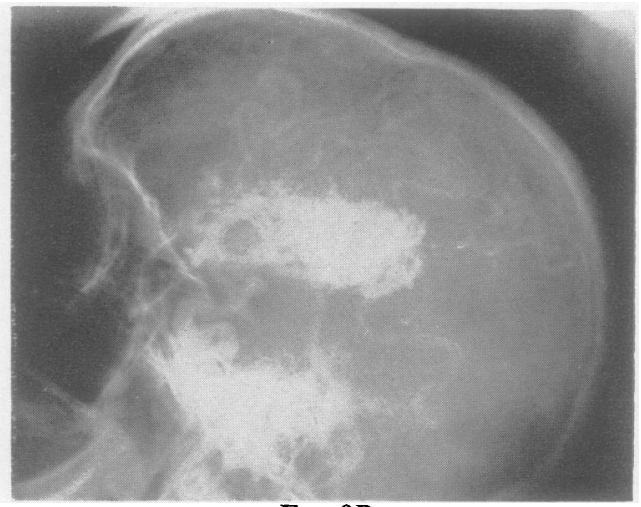

FiG. 9B

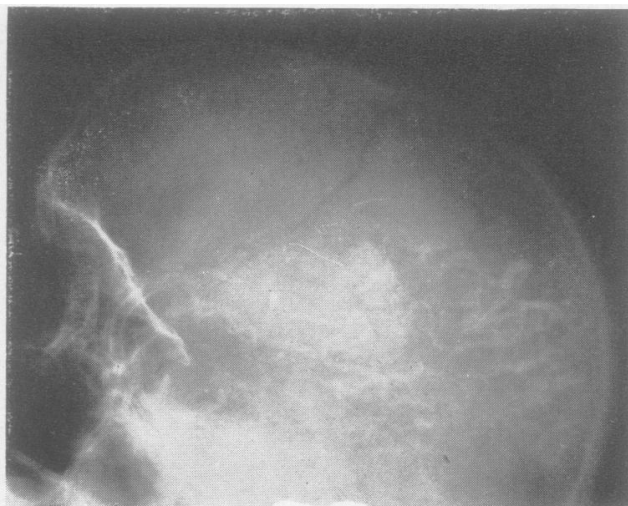

Fig. 9D

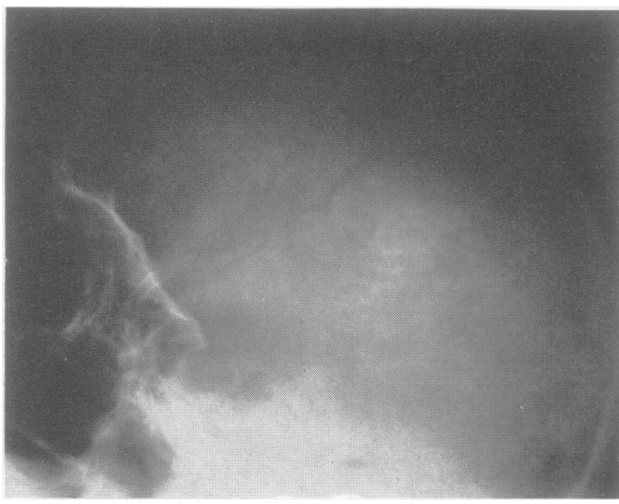

Fig. 9E

much less rapidity into those of the meningiomas. The duration of filling of tumour vessels may prove to be a valuable aid to the differential diagnosis of various types of neoplasm, but it is not possible to be definite because fistulae between arteries and veins may occasionally be found in certain meningiomas (Wickbom, 1948).

Arteriovenous Malformations.-Considerable assistance is given to the surgeon who intends to 
operate on arteriovenous malformations by an accurate knowledge of the afferent and efferent vessels to such a lesion. This enables them to be occluded in the correct order, and ensures that, so far as is possible, the difficulties of the operation are foreseen.

The circulation in certain arteriovenous malformations may be extremely rapid on account of the absence of capillary resistance, and the contrast may pass so quickly through these lesions that the arteries and veins are not seen separately in films obtained at the usual interval of 2 seconds (Wickbom, 1950). Furthermore, the whole of the malformation is not always filled at the same time or from a single source. A large lesion of this type is shown in Fig. 9. The serial angiograms of this patient were performed by the internal carotid and vertebral routes, and show an extensive arteriovenous malformation supplied from each of the three large cerebral arteries, with a gradual spread of the contrast substance into different parts of the lesion. The later frames from the vertebral series (Fig. 9E) show the development of a veil-like appearance caused by venous sacs, which often fill late in the series and are not always completely outlined in any one frame. The presence of such dilated venous channels greatly increases the difficulties of operation.

The complete film obtained from a patient with an unusual type of capillary angioma was illustrated in the preliminary report (Curtis, 1949). In this there was late filling of certain abnormal vessels which would not have been apparent in the routine series of three films. This lesion has not been confirmed histologically because the patient's epilepsy is adequately controlled by medical treatment and her symptoms do not warrant surgical exploration at present.

Aneurysms.-An interesting demonstration is provided, by means of rapid serial angiography, of the duration of filling of large saccular aneurysms of the carotid siphon which may retain the contrast substance after it has left the rest of the cerebral vessels (Fig.10).

Carotico-cavernous Fistulae. - The contrast substance passes so rapidly from artery to vein in these cases that, with our present machine, the serial

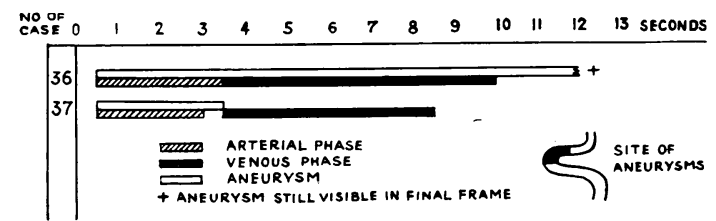

FIG. 10.-Duration of filling of two aneurysms situated on the carotid siphon. The diameter of the aneurysm in Case 36 was $2.8 \mathrm{~cm}$., and that in Case 37 was $1.0 \mathrm{~cm}$. Pooling of contrast occurs in the larger aneurysmal sac. frames do not succeed one another quickly enough to show the stages of its passage through the fistula. We have only used the rapid serial method in one case of this nature, but in this it did not appear to provide any particular advantage over the standard technique.

Thrombosis.-In two cases of thrombosis of the middle cerebral artery we observed a change in the extent of the territory supplied by the anterior cerebral artery (Fig. 11A), and clearly demonstrated that the ascending branches of the middle cerebral artery can in these circumstances be filled from above. In each of these cases the contrast filled the anterior cerebral artery and its named branches,

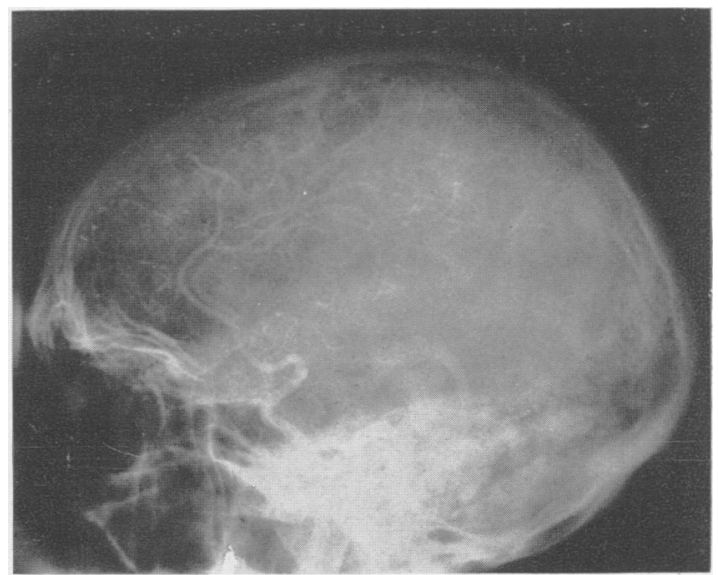

Fig. 11A

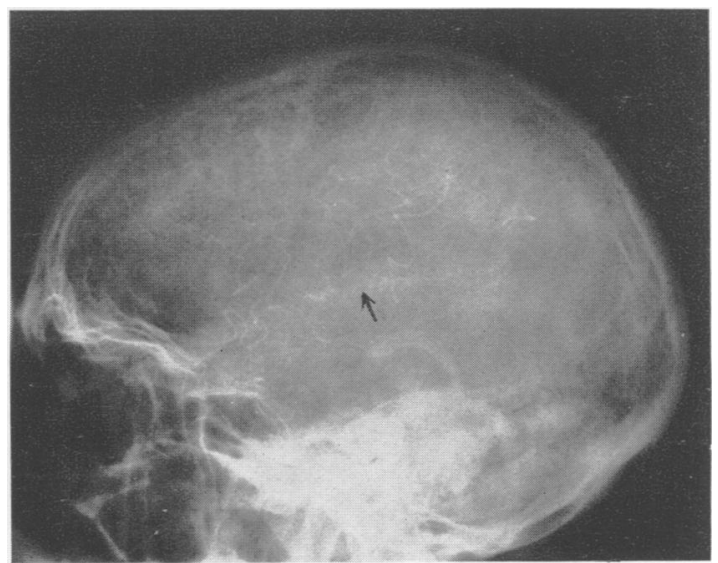

FIG. 11B

Fig. 11.-A. $3 \frac{1}{3}$ seconds. B. 4 seconds.

Consecutive frames from a serial angiogram of a case of middle cerebral thrombosis, showing how the contrast substance, in the absence of pressure in the reverse direction, flows through anastomotic channels into the territory normally supplied by the thrombosed vessel. Notice further filling of vessels $(\uparrow)$ in the second frame. 
and then flowed like a fountain over the surface of the hemisphere into the areas usually supplied by the middle cerebral artery. In the films the contrast could be clearly seen to pass from the small branches of the anterior cerebral artery into the corresponding branches of the middle cerebral artery, the retrograde filling of which gradually increased in the later frames (Fig. 11B). The serial angiogram of the case illustrated was repeated at the same session after blockage of the stellate ganglion with $1 \%$ procaine. No significant change was observed, but two and a half weeks had elapsed between the onset of the clinical symptoms and the interruption of the sympathetic pathways.

These cases show that the blood supply to areas affected by thrombosis can be restored not only by recanalization of thrombosed vessels, but also by the opening up of anastomotic channels to allow the passage of blood from other main arteries. These findings are also useful in angiographic diagnosis because, when the middle cerebral vessels are poorly filled, doubt often exists as to whether this is within normal limits or due to occlusion of small branches. If retrograde filling can be demonstrated, it is clear that there is in fact a thrombosis.

\section{Conclusions}

Rapid serial angiography is of considerable valuc as a research technique, and, when some of the variable factors are controlled, is likely to prove as useful in studying the physiology of the circulation of the brain as it has already proved to be in the study of the circulation of other organs. A modification of the machine described was used by Daniel and Prichard (1951) in their studies of the hepatic circulation. These workers used thorotrast for their contrast medium, and for the study of physiological conditions a less irritating substance than diodone must be used; we have therefore not attempted to collect material for the study of normal physiology from among our cases. The method of serial angiography is also of considerable educational value, since knowledge of the more complete picture of the passage of contrast substance through the cerebral vessels helps in the interpretation of other angiograms.

For clinical purposes there are certain circumstances in which rapid serial angiography may provide information which is not otherwise obtainable, although for routine work the standard technique of making three exposures at 2,4 , and 8 seconds has proved adequate. The method is of greatest use in studying the circulation of arteriovenous malformations, and may give the surgeon valuable information if surgical treatment of these lesions is contemplated. The second important indication for its use is when there is difficulty in the interpretation of angiograms obtained by the standard method; for example, when the possibility of malignant change in a tumour is open to question. Lastly, the demonstration of retrograde flow, in cases in which small vessels are thrombosed, may help in the diagnosis of these lesions.

\section{Summary}

The experience obtained with rapid serial angiography since the preliminary report was made in 1949 is reviewed.

Twenty-one normal serial angiograms are discussed with reference to the sequence and duration of filling of cerebral vessels during angiography.

The appearances of the choroidal plexus of the eye in rapid serial angiograms are described.

The uses of serial angiography in the diagnosis of tumours and in depicting arteriovenous malformations are considered.

Some observations are made on the course of the blood flow in cases of thrombosis of the middle cerebral artery.

Serial angiography should be used to supplement the information supplied by the standard (threefilm) technique, particularly in cases of vascular malformation and in conditions in which interpretation of the standard films is difficult. It may also be of assistance in some cases of cerebral thrombosis. The technique has great value for research and teaching purposes.

We acknowledge our indebtedness to Mr. J. B. Curtis who, with Dr. E. Schuster, O.B.E., was responsible for the development of the apparatus, and also took many of the angiograms we have reviewed. We are also grateful to Dr. D. G. Wyatt of the Nuffield Institute for Medical Research for measuring the irradiation, and to the Editor of the British Journal of Ophthalmology for permission to reproduce Figs. 5A and 5B.

\section{REFERENCES}

Curtis, J. B. (1949). Journal of Neurology, Neuro. surgery and Psychiatry, 12, 167.

(1951). Brit. J. Surg., 38, 295.

Daniel, P. M., and Prichard, M. M. L. (1951). J. Physiol., Lond., 112, 30.

Florey, H. (1925). Brain, 48, 43.

Forbes, H. S., and Wolff, H. G. (1928). Arch. Neurol. Psychiat., Chicago, 19, 1057.

Moniz, E. (1940). "Die Cerebrale Arteriographie und Phlebographie, "Handbuch der Neurologie" ed. Bumke, O., and Foerster, $O$. Berlin.

Schurr, P. H. (1951). Brit. J. Ophthal., 35, 473.

Trueta, J., Barclay, A. E., Daniel, P. M., Franklin, K. J., and Prichard, M. M. L. (1947). "Studies of the Renal Circulation." Oxford.

Wickbom, I. (1948). Acta. Radiol., Stockh. Suppl. 72. (1950). Ibid., 34, 385. 